\title{
Daily versus weekly azithromycin in cystic fibrosis patients
}

\author{
J. McCormack*, S. Bell\#, S. Senini*, K. Walmsley*, K. Patel*, C. Wainwright ${ }^{\pi}$, \\ D. Serisier ${ }^{+}$, M. Harris ${ }^{\S}$ and S. Bowler
}

ABSTRACT: Four randomised, placebo-controlled trials have previously documented the clinical benefits of azithromycin (AZM) in cystic fibrosis (CF) patients. The present study examined whether the beneficial effect of AZM is equivalent when administered daily or weekly.

A double-blind, randomised study was carried out in 208 CF patients aged 6-58 yrs who were assigned to AZM either $250 \mathrm{mg}$ daily $(n=103)$ or $1,200 \mathrm{mg}$ weekly $(n=105)$ for 6 months, with assessments at baseline and at 1, 3, 6 and 7 months. Patients were taken from five adult and children CF centres in South-east Queensland, Australia.

Equivalence was demonstrated between the two groups (daily versus weekly) with respect to improvements in lung function (forced expiratory volume in one second and forced vital capacity), C-reactive protein, days spent in hospital, admission rates and nutrition (body mass index, zscores) using $95 \%$ confidence intervals with a tolerance interval of $\pm 10 \%$.

In patients aged $<\mathbf{1 8}$ yrs the daily group had significantly better improvements in z-scores for height and weight after 6 months. In children, a nutritional advantage for daily administration was found. Gastro-intestinal adverse effects were more common with weekly therapy. Apart from these findings, daily and weekly administered azithromycin demonstrated similar outcomes for cystic fibrosis patients.

KEYWORDS: Azithromycin, cystic fibrosis, macrolides, Pseudomonas aeruginosa

$\mathrm{T}$ he idea of using macrolides in cystic fibrosis (CF) originated from a Japanese study, which demonstrated beneficial effects from erythromycin in diffuse panbronchiolitis, a disease with many similarities to CF [1]. To date four clinical trials have been carried out to demonstrate the beneficial effects of azithromycin (AZM) in patients with CF [2-5]. Despite differences in study populations (adult, paediatric and mixed), duration (3-12 months) and dosage, all studies demonstrated clinical improvement, which included significant improvement in lung function in three out of the four studies [2-4]. Interestingly, the longest study (12 months) failed to demonstrate significant improvement in spirometry in patients receiving AZM when compared with placebo. Reductions in hospitalisation and requirements for antibiotic therapy, decreased systemic markers of inflammation, and improvements in quality of life have been reported. Respiratory exacerbations and requirements for i.v. antibiotics were reduced in three of the studies $[2,3,5]$ Furthermore, AZM has been well tolerated in each of the four studies.

None of the studies demonstrated an apparent change in airway flora. There is ongoing concern about the long-term effects of macrolide therapy and whether such therapy may predispose to increased antimicrobial resistance of bacteria including Staphylococcus aureus, Streptococcus pneumoniae and Haemophilus influenzae [6, 7].

AZM has been shown to accumulate within tissues and neutrophils and this has been related to its prolonged biological effects [8-10]. These properties are different to other commonly prescribed macrolides, such as erythromycin, roxithromycin and clarithromycin, and may be responsible for the proposed anti-inflammatory effects of AZM demonstrated in CF patients. In a recent study examining the pharmacokinetics of AZM in plasma, neutrophils and sputum in CF patients, WiLms et al. [11] demonstrated marked accumulation of AZM in neutrophils with a mean half-life of 12 days. The concentration of AZM in sputum was also very high and still detectable 10 days after the last dose was administered. The authors suggested that this long half-life and persistence in sputum may allow less frequent dosing (e.g. once a week) in patients with $\mathrm{CF}$, which may encourage adherence.

Many questions remain regarding the therapeutic use of AZM and other macrolides in patients with $\mathrm{CF}$, including: dosing regimens (dose,

\section{AFFILIATIONS}

${ }^{*}$ Dept of Medicine and Infectious Diseases, and

${ }^{+}$Adult Cystic Fibrosis Unit, Mater Adult Hospital,

${ }^{\text {\#} A d u l t ~ C y s t i c ~ F i b r o s i s ~ C e n t r e, ~ T h e ~}$ Prince Charles Hospital,

"Dept of Paediatrics and Child Health, Royal Children's Hospital, and

${ }^{\S}$ Mater Children's Hospital, University of Queensland, South Brisbane, Queensland, Australia.

CORRESPONDENCE

J. McCormack

Dept of Medicine and Infectious Diseases

University of Queensland Mater Adult Hospital

South Brisbane

4101

Queensland

Australia

Fax: 61738401548

E-mail: jmccorma@mater.org.au

Received:

December 142006

Accepted after revision:

May 192007

\section{SUPPORT STATEMENT}

The study was supported by the Australian Cystic Fibrosis Research Trust (Sydney, Australia)

STATEMENT OF INTEREST

A statement of interest for this study can be found at

www.erj.ersjournals.com/misc/ statements.shtml 
frequency and duration); who should be treated; whether other macrolides are effective; and the long-term benefits and risks of continued AZM therapy [12]. The major objective of the present study was to determine the clinical effects of AZM administered weekly in children and adults with CF using a parallel group, randomised double-blind study design of 6 months' duration. The study was powered to determine whether weekly and daily AZM were equivalent in their effect on forced expiratory volume in one second (FEV1) \% predicted.

\section{METHODS}

\section{Design}

A double-blind randomised comparative study of $250 \mathrm{mg}$ daily versus 1,200 mg weekly AZM in adults and children with $\mathrm{CF}$ in five Australian centres was carried out. Eligibility criteria for recruitment were: 1) age $\geqslant 6 \mathrm{yrs}$; 2) weight $\geqslant 25 \mathrm{~kg} ; 3$ ) clinical stability, defined as patients experiencing lung function (FEV1) at $\geqslant 90 \%$ of the mean outpatient FEV1 recorded over the previous 6 months, with $\geqslant 2$ weeks since completion of last i.v./oral antibiotics for a pulmonary exacerbation; and 4) no concurrent change in medication. Patients were recruited irrespective of whether or not they were infected with Pseudomonas aeruginosa. Exclusion criteria included: 1) inability to provide informed consent; 2) known allergy to macrolides; 3) macrolide therapy in the previous 8 weeks; 4) severe liver disease (defined as proven cirrhosis and/or portal hypertension); 5) non-tuberculous mycobacteria isolated in sputum in the previous $2 \mathrm{yrs}$; 6) commencement of mucolytic or antiinflammatory therapy that had not been part of therapy prior to trial enrolment; 7) concomitant use of drugs contraindicated in patients taking macrolides (digoxin, cyclosporine, terfenadine, zidovidine or ergotamines); 8) transplant recipient patients; 9) patients who were pregnant, their partner was pregnant or they intended to get pregnant in the following 6 months; and 10) active involvement in another clinical trial. Usual concomitant therapy, including physiotherapy, maintenance antibiotics, inhaled medications and pancreatic and vitamin supplements, continued throughout the trial and was monitored by the clinic physicians.

Ethical approval was granted at each of the participating hospitals. After written informed consent by patients, or parents/guardians for patients aged $<18 \mathrm{yrs}$, patients were randomised to receive AZM daily or once a week for 6 months.

\section{Randomisation}

Randomisation was performed by the hospital pharmacy independently of trial staff prior to study commencement. Randomisation occurred in blocks of 10 patients and was stratified for sex and illness severity (FEV1 <40, 40-70 and $>70 \%$ pred). There was further stratification by age in adults [13] and height in children [14] to ensure equal representation across treatments.

\section{Allocation generation}

A randomisation schedule was generated by the Mater Hospital Pharmacy (South Brisbane, Queensland, Australia) for use at all centres. A Microsoft Excel program was used to randomly generate numbers, and randomly assign dosing regimes to patient numbers in blocks of 10 . Trial staff designated each patient the next available randomisation number in their stratification group from the randomisation schedule. Patients presented to the pharmacy with their allocated randomisation number and were provided with treatment from group $\mathrm{A}$ or $\mathrm{B}$, according to the master randomisation schedule code held by the pharmacy.

\section{Allocation concealment}

The two treatment doses were concealed in identical capsules manufactured by Mater Hospital Pharmacy and presented in blister packs. Blister packs were labelled with code " $\mathrm{A}$ " or " $\mathrm{B}$ " to ensure pharmacy staff supplying the patients with their study treatment were also blinded to the treatment allocation. This label was removed prior to the pack being provided to the patient. All patients were required to take three capsules on one set day of the week, comprising either the entire weekly dose for the weekly treatment group, or one daily capsule complimented with two placebos (lactose) in the daily treatment group. All capsules looked and tasted the same. The daily group continued with one 250-mg capsule per day for the remaining 6 days, while intercurrent doses in the onceweekly regimen consisted of a placebo. Unblinding occurred subsequent to statistical analysis.

\section{Outcome measures}

Patients were assessed at baseline, after 1, 3 and 6 months of treatment and at 1 month post-treatment. Assessments at each time-point included the primary outcome of patient lung function (FEV1 as measured by spirometry). Spirometry was performed in specialised respiratory function laboratories according to the American Thoracic Society (ATS) criteria [15] and the percentage predicted was calculated using specific reference ranges for adults [13] and children [14]. A small number of outreach patients were assessed using a portable spirometer (Microlab 3500; Micro Medical Ltd, Kent, UK) provided by the Mater Children's Hospital Respiratory Laboratory, which was regularly calibrated using a 3-L calibration syringe (Hans Rudolph Inc., Kansas City, MI, USA).

Secondary outcomes were also measured at each assessment and included forced vital capacity (FVC; measured by spirometry), percentage of patients admitted, mean days spent in hospital for respiratory indications, mean number of admissions for respiratory indications, nutritional status via body mass index (BMI) for adults ( $\geqslant 18$ yrs) and height, weight and BMI standardised z-scores for children and adolescent patients ( $\leqslant 18$ yrs old). Serum liver enzymes aspartate aminotransferase, alanine aminotransferase and the inflammatory mediator C-reactive protein (CRP) were measured by the Queensland Health Pathology Service (Brisbane, Queensland, Australia) using nephelometry. Serological evidence of infection with Legionella pneumophilia or Mycoplasma pneumoniae at baseline and after 6 months of treatment was sought to determine whether clinical improvement may be attributable to treatment of organisms susceptible to AZM.

Acute respiratory exacerbations were treated as usual by attending physicians with admission to hospital for increased respiratory symptoms when appropriate. AZM treatment was continued throughout these periods. Formal criteria for the definition of respiratory exacerbations were not utilised in the present trial. 
Where possible, an expectorated sputum sample was collected at each assessment and cultured by standard techniques for typical pathogens, including $P$. aeruginosa, $H$. influenzae, $S$. aureus and Burkholderia cepacia [16]. Throat swabs were collected to detect the presence of $S$. pneumoniae. AZM sensitivity testing of $S$. aureus isolates cultured before, during and after therapy was also performed [17].

Disc diffusion (Haemophilus spp.) and VITEK susceptibility panels (S. aureus) in an automated system (bioMérieux, Marcy l'Etoile, France) were used to determine antibiotic susceptibility. Clarithromycin was used to test macrolide susceptibility of Haemophilus spp. The National Committee for Clinical Laboratory Standards' breakpoints were used to determine susceptibility of the isolates, as has been previously applied by PHAFF et al. [7]. According to these criteria, S. aureus with erythromycin minimal inhibitory concentration (MIC) of $\geqslant 8 \mathrm{mg} \cdot \mathrm{L}^{-1}$ and Haemophilus spp. with clarithromycin disc zone $\geqslant 13 \mathrm{~mm}$, corresponding to $\mathrm{MIC} \geqslant 32 \mathrm{mg} \cdot \mathrm{L}^{-1}$, are classified as resistant.

Quality of life (QoL) was assessed using the disease-specific QoL questionnaires: Version 1 Cystic Fibrosis Questionnaire (CFQ) for either adults and adolescents $>14$ yrs old, or child and parent versions for younger children (researcher administered for patients 6-11 yrs old and self-administered for those aged 12-13 yrs).

\section{Statistical analysis and sample size}

The primary study outcome was a change from baseline in FEV1 \% pred. Additional analyses were conducted for secondary outcomes of change in FVC, CRP, nutritional status and time to first hospitalisation for exacerbation. Further secondary outcomes included change in number of admissions, length of stay and proportion of patients requiring hospital admissions for respiratory exacerbations relative to the 6-month period immediately prior to randomisation. Response heterogeneity was determined through sub-analyses of pulmonary level of illness severity (FEV1 $<40,40-70$ and $>70 \%$ pred), and distinct age group analyses for patients $<14$ yrs old and $\geqslant 14$ yrs old to assess treatment responses in lung function, $\mathrm{CRP}$, number of admissions and length of stay.

In a previous study, involving 30 patients per arm, an SD of $22.5 \%$ was observed for a change in the primary outcome (FEV1 \% pred) [2]. Based on this SD, the current authors originally calculated that a sample size of 92 patients per arm would be required to show equivalence in the primary outcome of change in FEV1 \% pred with a tolerable interval of $\pm 10 \%$ with $85 \%$ power and a $5 \%$ significance level. However, the SD achieved in the current study was $20.04 \%$; therefore, it was calculated that a sample size of 77 patients per arm was required to show equivalence. The present sample size calculations were based on the primary rather than secondary outcomes. These numbers were exceeded out of concerns regarding attrition and incomplete data. Analysis of the data was carried out using an intent-to-treat principle. To begin with, the demographic factors between the group that withdrew from the study and the remaining cohort were compared. No significant differences were found between any demographic factors (table 1), implying that bias was not introduced by the dropouts. Subsequently, generalised

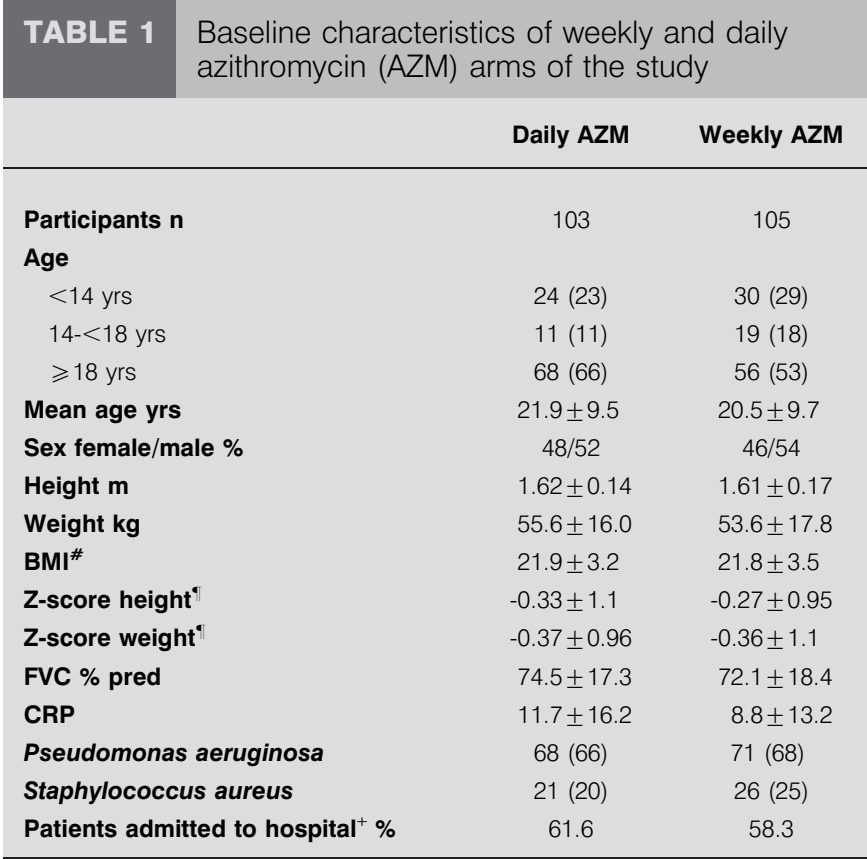

Data are presented as $n(\%)$ or mean $\pm S D$, unless otherwise stated. BMI: body mass index; FVC: forced vital capacity; \% pred: \% predicted; CRP: Creactive protein. ${ }^{*}$ : patients aged $\geqslant 18 \mathrm{yrs}^{\circ}{ }^{\circ}$ : patients aged $<18 \mathrm{yrs}$; ${ }^{+}$: i.v. antibiotics in 6 months prior to the study.

estimating equations were used because of their ability to analyse correlated longitudinal data with missing values, assuming that the data was missing completely at random. All recruited and randomised participants were included in the analyses. All analyses reported significance at the $p<0.05$ level (two-tailed). At the bivariate level, analysis involved Fisher's exact test to examine the significance of the association between independent categorical variables. Assumption of normality was tested via the Shapiro and Francia Test, and where the assumption could not be met, data were transformed logarithmically or via square root transformation for analysis. When data could not be transformed to represent a normal distribution, the Wilcoxon Mann-Whitney rank sum test was employed. Within-group mean changes from baseline and between-group comparisons were calculated using generalised estimating equation analyses with workable exchangeable correlation structure. Assessment of microbiological changes over the course of treatment employed the $\mathrm{K}$ coefficient to measure the agreement between baseline and 6month treatment scores. With only two discrete levels of outcome, the Exact McNemar test was used to test significance of changes over the treatment period.

\section{RESULTS}

In total, $208 \mathrm{CF}$ patients (47\% female) with a mean (range) age of $21.2(6-58)$ yrs were recruited from a total of 379 patients assessed for eligibility at five CF centres (two adult, two paediatric and one mixed; fig. 1). The baseline characteristics of the patients in each arm are described in table 1 . There were no differences in any of the parameters examined. Out of the patients who completed the 6 month trial, 26 started in the 


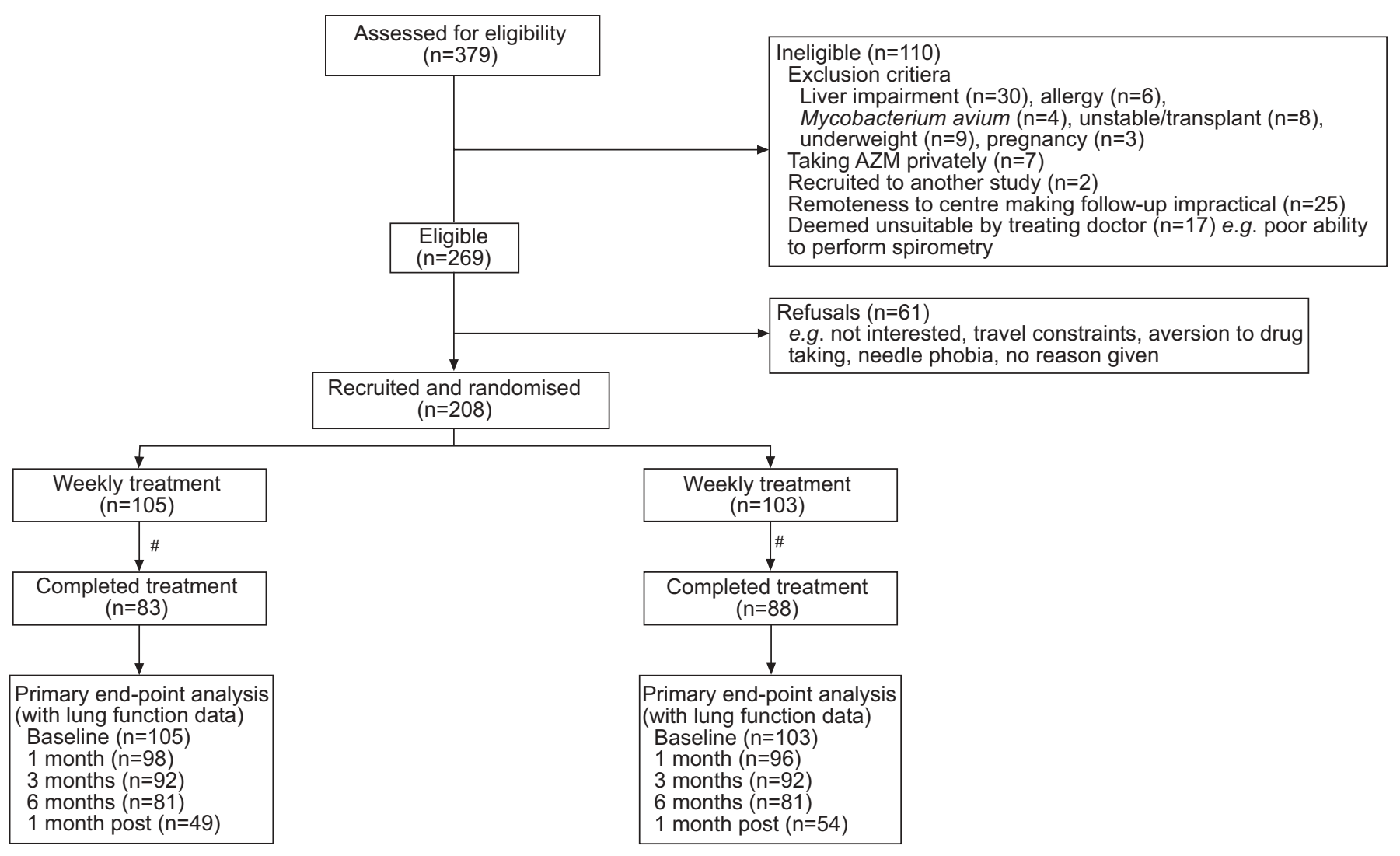

FIGURE 1. Flow diagram showing the trial profile. AZM: azithromycin. ${ }^{\#}$ : during the trial a total of 37 (18\%) subjects withdrew due to: choice to withdraw or inability to attend assessments ( $n=14)$; loss to follow-up $(n=9)$; abdominal symptoms, nausea, pancreatitis, anorexia and weight loss, diarrhoea, vomiting ( $n=9)$; ocular surface irritation $(n=1)$; lung transplant $(n=2)$, mood swings $(n=1)$; or photosensitivity $(n=1)$.

summer, 51 in autumn, 37 in winter and 56 in the spring, but the starting season was equivalent for the weekly and daily arms $(p=0.42)$. Of the 208 enrolled patients, 26 were located at remote sites outside Brisbane (Australia) but their care was coordinated by one of the five centres.

Tables 2-4 show the changes in FEV1\% pred from baseline to 1, 3, 6 and 7 months. Comparative values are shown for patients aged $>14 \mathrm{yrs}$ and $<14 \mathrm{yrs}$ old. There was no difference between the weekly and daily groups at any of the time-points. When all patients were included in the analysis of changes from baseline, the two treatment groups were equivalent to an interval of $\pm 3.2 \%$. During the treatment period, adults and adolescents $\geqslant 14$ yrs old and $<14$ yrs old proved equivalent to \pm 2.4 and $\pm 8.7 \%$, respectively. There was also no difference between the two groups when the relative change from baseline in FEV1 \% pred was compared, as shown in figure 2.

The FVC values also showed no differences between patients in the weekly or daily arms for adults or children (data not shown).

The changes in CRP are shown in table 5. The decrease in CRP in the daily group was larger than the weekly group at 3 and 6 months ( $p=0.04$ and 0.02 , respectively). There were no such differences at 1 or 7 months $(\mathrm{p}=0.31$ and 0.25 , respectively).
The percentage of patients admitted before and during the study period, the number of days spent in hospital, the number of admissions and the time to first exacerbation are shown in table 6 . In the 6 months prior to the study, respiratory admission numbers were greater in the daily group but hospitalisation days were equivalent in both groups. No difference was demonstrated between the weekly and daily groups for hospital days, admission numbers or time to respiratory hospitalisation during the course of the study.

When changes in nutritional status in the two groups were examined there were no differences in the change in BMI between the weekly and daily groups in the patients aged $\geqslant 18$ yrs after 6 months of treatment $(p=0.88)$. However, in patients $<18$ yrs old, the daily group had significantly better improvements in z-scores for height and weight at 6 months (fig. 3) but this was not the case for BMI z-scores.

Health-related QoL modalities were equivalent in the weekly and daily groups at baseline (data not shown). For patients who were $\geqslant 14$ yrs of age, there were significant differences between groups in favour of weekly AZM in the physical domain at 6 months compared with baseline ( +8.2 points; $p=0.02$ ). There were no differences in any of the other domains between groups at the end of the study. For patients aged $<14$ yrs, the health domain of the CFQ for parents (parents' responses) was significantly higher at 6 months for the daily 


\begin{tabular}{|c|c|c|c|c|c|}
\hline \multirow[t]{2}{*}{ TABLE 2} & \multicolumn{5}{|c|}{$\begin{array}{l}\text { Change from baseline in forced expiratory } \\
\text { volume on one second ( } \% \text { pred) over } 7 \text { months } \\
\text { and by treatment group in all patients }{ }^{\#}\end{array}$} \\
\hline & Baseline & 1 month & 3 months & 6 months & 7 months \\
\hline Daily $^{+}$ & $56.4 \pm 19.6$ & $2.8 \pm 0.7^{*}$ & $3.5 \pm 0.8^{*}$ & $3.1 \pm 0.8^{*}$ & $1.2 \pm 1.0$ \\
\hline Weekly ${ }^{\S}$ & $57.0 \pm 20.6$ & $2.4 \pm 0.8^{*}$ & $2.3 \pm 0.7^{*}$ & $2.4 \pm 1.0^{*}$ & $1.2 \pm 1.2$ \\
\hline $\begin{array}{l}\text { Mean } \\
\text { difference }^{f}\end{array}$ & 0.6 & -0.4 & -1.2 & -0.7 & 0.0 \\
\hline $95 \% \mathrm{Cl}$ & $-5.0-6.1$ & $-1.7-2.4$ & $-0.9-3.3$ & $-1.7-3.2$ & $-3.0-3.0$ \\
\hline p-value $\# \#$ & & 0.74 & 0.27 & 0.58 & 1.00 \\
\hline \multicolumn{6}{|c|}{$\begin{array}{l}\text { Data are presented as mean } \pm \mathrm{SD} \text {, unless otherwise stated. Cl: confidence } \\
\text { interval. }{ }^{\#:} n=208 ;{ }^{\uparrow}: \text { change compared with baseline; }{ }^{+}: n=103 ;{ }^{s}: n=105 ; \\
\text { mean difference between groups (daily versus weekly); }{ }^{\# \#: ~} p \text {-values relate to } \\
\text { mean difference between groups (daily versus weekly); }{ }^{*}: \text { significant difference } \\
(p<0.05) \text { from baseline. }\end{array}$} \\
\hline
\end{tabular}

AZM (+15.6 points; $\mathrm{p}=0.02)$ but there were no differences in any other domain. For children (children' responses) no differences were found at 6 months compared with baseline in either group for any of the CFQ domains.

$P$. aeruginosa was found in $66 \%$ of patients' sputum on recruitment, with no difference between the weekly and daily treatment group. There was no change in this percentage over the study duration.

S. aureus was identified in sputum from 47 out of 181 patients at baseline and from 22 out of 141 patients at 6 months. There was no significant difference in isolation rates between the weekly and daily groups. The number of patients in the weekly and daily groups who had isolates of $S$. aureus at baseline and 6 months were examined, and no difference was found between the two. In 24 patients with isolates at baseline and at least one other trial time-point, $14 \mathrm{~S}$. aureus isolates were sensitive to AZM, five of which became resistant over the course of treatment. Of these five isolates, two were in the

\begin{tabular}{|c|c|c|c|c|c|}
\hline \multirow[t]{2}{*}{ TABLE 3} & \multicolumn{5}{|c|}{$\begin{array}{l}\text { Change from baseline in forced expiratory volume } \\
\text { in one second (\% pred) over } 7 \text { months and by } \\
\text { treatment group in patients aged }<14 \mathrm{yrs}^{\#}\end{array}$} \\
\hline & Baseline & 1 month & 3 months & 6 months & 7 months \\
\hline Daily $^{+}$ & $65.1 \pm 18.3$ & $2.5 \pm 1.5$ & $4.6 \pm 2.1^{*}$ & $5.3 \pm 1.95^{\star}$ & $3.7 \pm 3.15$ \\
\hline Weekly ${ }^{\S}$ & $66.5 \pm 15.2$ & $2.2 \pm 1.8$ & $2.8 \pm 1.8$ & $2.7 \pm 2.41$ & $-0.6 \pm 3.46$ \\
\hline $\begin{array}{l}\text { Mean } \\
\quad \text { difference }\end{array}$ & +1.4 & -0.3 & -1.8 & -2.6 & -4. 3 \\
\hline $95 \% \mathrm{Cl}$ & $-7.74-10.53$ & $-4.33-4.93$ & $-3.7-7.32$ & $-3.47-8.67$ & $-4.94-13.4$ \\
\hline p-value $\# \#$ & & 0.90 & 0.52 & 0.40 & 0.37 \\
\hline
\end{tabular}

Data are presented as mean $\pm \mathrm{SD}$, unless otherwise stated. $\mathrm{Cl}$ : confidence interval. ${ }^{\#}: n=54 ;{ }^{\natural}$ : change compared with baseline; ${ }^{+}: n=24 ;^{\S}: n=30 ;$; : mean

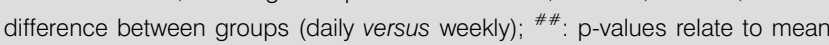
difference between groups (daily versus weekly); *: significant difference $(p<0.05)$ from baseline.

\begin{tabular}{|c|c|c|c|c|c|}
\hline \multirow[t]{2}{*}{ TABLE 4} & \multicolumn{5}{|c|}{$\begin{array}{l}\text { Change from baseline in forced expiratory volume } \\
\text { in one second ( } \% \text { pred) over } 7 \text { months and by } \\
\text { treatment group in patients aged } \geqslant 14 \mathrm{yrs}^{\#}\end{array}$} \\
\hline & Baseline & 1 month & 3 months & 6 months & 7 months \\
\hline Daily $^{+}$ & $53.8 \pm 19.3$ & $2.9 \pm 0.8^{*}$ & $3.2 \pm 0.8^{*}$ & $2.4 \pm 0.8^{\star}$ & $0.4-0.9$ \\
\hline Weekly ${ }^{\S}$ & $53.2 \pm 21.3$ & $2.5 \pm 0.8^{*}$ & $2.1 \pm 0.7^{*}$ & $2.3 \pm 0.9^{*}$ & $1.8-0.9^{*}$ \\
\hline $\begin{array}{l}\text { Mean } \\
\text { difference }\end{array}$ & -0.6 & -0.4 & -1.1 & -0.1 & +1.4 \\
\hline $95 \% \mathrm{Cl}$ & $-5.87-7.05$ & $-1.89-2.54$ & $-1.07-3.17$ & $-2.28-2.37$ & $-3.9-1.08$ \\
\hline p-value ${ }^{\# \#}$ & & 0.77 & 0.33 & 0.97 & 0.26 \\
\hline
\end{tabular}

Data are presented as mean $\pm \mathrm{SD}$, unless otherwise stated. $\mathrm{Cl}$ : confidence interval. ${ }^{\#}: n=154 ;{ }^{\bullet}$ : change compared with baseline; ${ }^{+}: n=79 ;{ }^{~}{ }^{5}: n=75 ;{ }^{f}$ mean difference between groups (daily versus weekly); ${ }^{\# \#}: p$-values relate to mean difference between groups (daily versus weekly); *: significant difference $(p<0.05)$ from baseline.

weekly group and three were in the daily group. There was no difference in the changes in FEV1 between these five patients and the other patients. There were no S. pneumoniae isolates in any patient at commencement or during the study.

There was no difference between groups at baseline in isolation rate of $H$. influenzae, with two isolates in the weekly and one in the daily groups, and no isolates after 6 months of treatment.

B. cepacia complex was isolated from five out of the 91 patients tested at baseline from the weekly treatment group, and one out of the 90 patients from the daily treatment group. At the end of 6 months, the isolation rates were three out of 69 and one out of 78 for the weekly and daily groups, respectively. After 6 months of treatment, no difference was found between the groups in the number of isolates of $B$. cepacia complex $(p=0.46)$ and no patients had acquired this infection during the course of treatment.

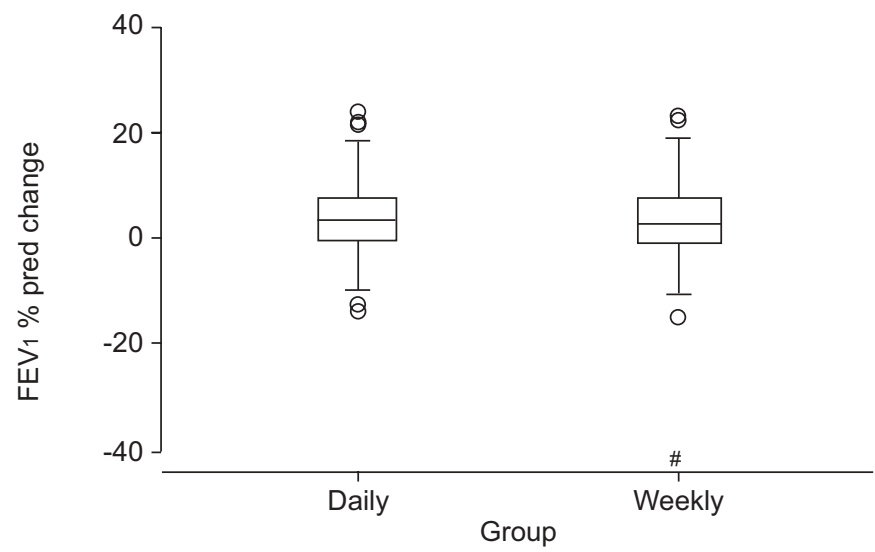

FIGURE 2. Change in forced expiratory volume in one second (FEV1) between 6 months and baseline after 6 months of treatment. The error bars represent the median with the smallest and largest observations within 1.5 times the interquartile range (IQR). $\bigcirc$ : outliers between 1.5 and 3 times IQR from the quartiles. ${ }^{\#}$ : data point $>3$ times IQR from the quartiles. \% pred: \% predicted. 


\begin{tabular}{|c|c|c|c|c|c|}
\hline \multirow[t]{2}{*}{ TABLE 5} & \multicolumn{5}{|c|}{$\begin{array}{l}\text { Change from baseline in C-reactive protein over } \\
7 \text { months and by treatment group }\end{array}$} \\
\hline & Baseline & 1 month $^{\#}$ & 3 months ${ }^{\#}$ & 6 months ${ }^{\#}$ & 7 months ${ }^{\#}$ \\
\hline Daily & $11.7 \pm 16.2$ & $-4.7 \pm 1.7^{\star}$ & $-6.5 \pm 1.6^{*}$ & $-5.8 \pm 1.6^{*}$ & $-3.0 \pm 2.1$ \\
\hline Weekly & $8.8 \pm 13.2$ & $-2.6 \pm 1.2^{*}$ & $-1.3 \pm 2.0$ & $-0.7 \pm 1.3$ & $-0.1 \pm 1.3$ \\
\hline $\begin{array}{l}\text { Mean } \\
\text { difference }\end{array}$ & -2.9 & +2.1 & +5.2 & +5.1 & +2.9 \\
\hline $95 \% \mathrm{Cl}$ & $-6.3-2.0$ & $-6.3-2.0$ & $-10.2--0.1$ & $-9.3--1.0$ & $-7.7-2.0$ \\
\hline p-value ${ }^{+}$ & & 0.31 & 0.04 & 0.02 & 0.25 \\
\hline
\end{tabular}

Data are presented as mean $\pm \mathrm{SD}$, unless otherwise stated. Cl: confidence

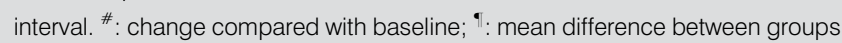
(daily versus weekly); ${ }^{+}: \mathrm{p}$-values relate to mean difference between groups (daily versus weekly); *: significant difference $(p<0.05)$ from baseline.

The adverse events of the study are listed in table 7 . The most obvious difference between the two groups was the higher rate of gastro-intestinal effects in patients taking weekly AZM. It is likely that this was attributable to the larger dose. At 6 months five participants had dropped out of the daily treatment arm and 12 had dropped out of the weekly treatment arm (odds ratio $0.40,95 \%$ confidence interval $0.134-1.166)$. The most common adverse event involved nausea (daily $n=2$, weekly $n=11$ ), followed by diarrhoea (daily $n=3$, weekly $n=8$ ) and vomiting (daily $n=0$, weekly $n=5$ ).

The total cost of AZM for 6 months was AU\$407 and AU\$622 for weekly and daily use, respectively; a factor of 0.65 . The cost of AZM was calculated based on the distribution charge from the Mater Hospital Pharmacy and did not include any ancillary charges.

\section{DISCUSSION}

The benefits of AZM in CF patients has been clearly demonstrated in four placebo-controlled, randomised controlled trials (RCTs) [2-5], as summarised in table 8. Further trials are needed to define optimal usage of AZM in CF. The previous studies have involved AZM administration daily or three times a week. The present study examined the potential for use of weekly dosing over a 6-month period in children and adults with $\mathrm{CF}$ with ages ranging 6-58 yrs. The weekly dose was 1,200 mg per

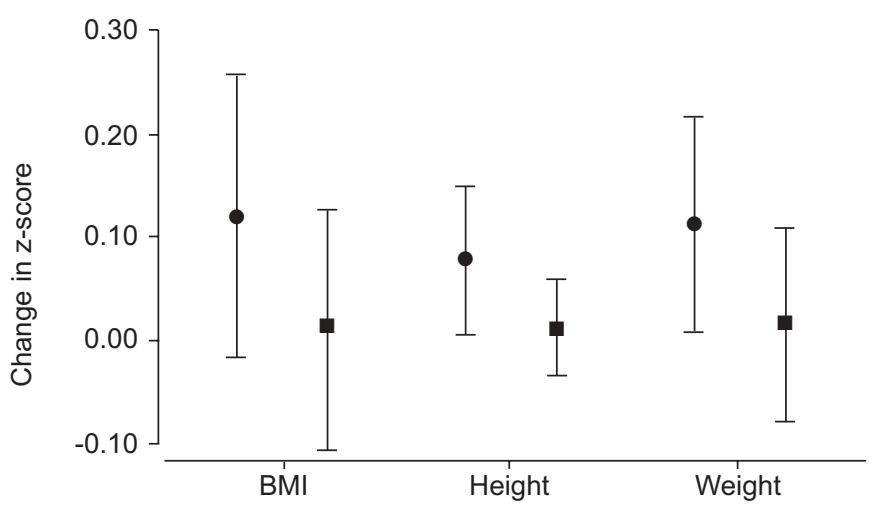

FIGURE 3. Mean change in z-scores from baseline to 6 months in body mass index (BMI), height and weight in subjects aged $<18$ yrs. The error bars represent 95\% confidence intervals.

: daily azithromycin;

: weekly azithromycin.

week; this was selected because it is the regimen widely used in HIV/AIDS patients with low CD4 lymphocyte counts for prophylaxis against Mycobacterium avium infections and has been shown to be tolerable in that group [18].

At baseline, there were no significant differences between the characteristics of the two groups. The current study demonstrated equivalence between weekly and daily administered AZM for the primary end-point, FEV1 \% pred. The magnitude of change in FEV1 for the daily therapy was similar to that seen at 3 months in an earlier study and in two out of the three previously published RCTs. Notably, the most recent and longest (12 months) RCT failed to demonstrate an improvement in spirometry [5]. Secondary end-points, including hospitalisation for respiratory treatments, i.v. antibiotic rates, time to hospitalisation and QoL, were equivalent between treatment arms in the present study.

The current authors could not demonstrate any difference between those who received weekly or daily AZM. However, encouragingly, the effect on hospitalisation seen in earlier studies [2, 4] was also evident in the current study and was seen in both treatment arms. The number of days spent in hospital may be an important contributor to a patient's QoL and has important social and financial implications for both the patient and health systems. Whilst protocol definition for

TABLE 6 Patients admitted, time spent in hospital, number of admissions and time to first exacerbation in study population

\begin{tabular}{|c|c|c|c|c|c|c|c|c|c|c|}
\hline & \multicolumn{3}{|c|}{ Patients admitted \% } & \multicolumn{3}{|c|}{ Mean days in hospital } & \multicolumn{3}{|c|}{ Mean number of admissions } & $\begin{array}{c}\begin{array}{c}\text { Mean days } \\
\text { to first } \\
\text { exacerbatior }\end{array} \\
\text { Trial }\end{array}$ \\
\hline Daily & 62 & 32 & $<0.0001$ & $15.5 \pm 18.9$ & $6.5 \pm 12.1$ & $<0.0001$ & $1.3 \pm 1.3$ & $0.6 \pm 1.0$ & $<0.0001$ & $70.0 \pm 44.8$ \\
\hline
\end{tabular}

\#: 6 months immediately prior to study commencement; ${ }^{\uparrow}: \mathrm{p}$-values relate to difference within group over time; ${ }^{+}: \mathrm{p}$-values relate to differences between groups (daily versus weekly). 
TABLE 7 Adverse events by treatment arm

\begin{tabular}{|c|c|c|c|}
\hline Total adverse events & $24 / 103$ & $34 / 105$ & 0.27 \\
\hline Frequency of four-fold increase from baseline in ALT & $4 / 103$ & $1 / 104$ & 0.21 \\
\hline Frequency of four-fold increase from baseline in AST & 2/103 & $1 / 103$ & 1.00 \\
\hline
\end{tabular}

Data are presented as n/total number of subjects, unless otherwise stated. ALT: alanine aminotransferase; AST: aspartate aminotransferase. ${ }^{\# \text { : }}$ gastro-intestinal adverse events of diarrhoea, nausea, abdominal symptoms, vomiting, constipation, pancreatitis, anorexia and weight loss; nongastro-intestinal adverse events of thrush, haemoptysis, headache, photosensitivity, rash, ocular surface irritation, dehydration, nightmares, difficulty concentrating, mood swings, hearing muffled, dry skin and hair, and pneumothorax.

pulmonary exacerbations were not included in the present study, a surrogate marker, hospitalisation for pulmonary indications, was used and was not different between the two treatment arms. In an earlier study [2] and a study from the USA [4] there was a reduction in the number of days spent in hospital for patients who received AZM. No such reduction was observed in a study from the UK [3]. Therapeutic modalities that can reduce days spent in hospital are worthy of detailed evaluation, particularly in long-term studies.

The mechanism of action of macrolides in CF is not yet established; however, anti-inflammatory and antimicrobial effects have been proposed. The evidence favouring each of these mechanisms has previously been summarised [12]. The study by WOLTER et al. [2] demonstrated that patients treated with AZM had a reduction in their mean CRP values in comparison with patients given placebo. The current study found differences between the weekly and daily treated patients at 3 and 6 months, but not at 1 and 7 months (table 5). These findings may suggest that the beneficial effects of AZM take several weeks to be established and are lost within a month of drug cessation whether the treatment is taken daily or weekly.

Adverse events were similar overall between treatment groups but there were increased gastro-intestinal adverse events in the weekly group. In the current study there were no differences in BMI z-scores between daily and weekly treated patients in the adult population (fig. 3), but better improvements were noted in the height and weight $\mathrm{z}$-scores in the daily group in patients $<18 \mathrm{yrs}$ old. The magnitude of the increase in growth parameters is relatively small, although this needs to be considered in light of a short duration study (6 months) and is likely to be an important clinical measure to determine benefit from AZM in younger patients with $\mathrm{CF}$. The latter findings were unexpected; however, they may, in part, relate to the increased gastro-intestinal adverse effects in the patients receiving AZM weekly. If confirmed in other studies, this may have implications for future management. In the interim, weekly treatment should probably be avoided in younger patients. Assessment of changes in nutritional status has been

TABLE 8 Study design and clinical outcomes in azithromycin in cystic fibrosis randomised controlled trials

\begin{tabular}{|c|c|c|c|c|}
\hline \multicolumn{5}{|l|}{ Study design } \\
\hline Patients n & 60 & 41 & 185 & 82 \\
\hline Population & Adult & Paediatric & Adult/paediatric & Adult/paediatric \\
\hline Duration months & 3 & $15^{\#}$ & 6 & 12 \\
\hline Baseline FEV1 \% pred & 57 & 61 & 70 & 85 \\
\hline Relative $\%$ change in FEV $1 \%$ pred & +3.6 & +5.4 & +6.2 & -4.3 \\
\hline Hospital days & $\downarrow$ & $\leftrightarrow$ & $\downarrow$ & NR \\
\hline Intravenous antibiotic days & $\downarrow$ & $\leftrightarrow^{+}$ & $\downarrow$ & $\downarrow$ \\
\hline Inflammatory markers & $\downarrow$ & $\leftrightarrow$ & $\downarrow$ & NR \\
\hline
\end{tabular}

FEV1: forced expiratory volume in one second; \% pred: \% predicted; QoL: quality of life; MWF: Monday, Wednesday, Friday (days of administration); NR: not reported; $\downarrow$ decreased; $\leftrightarrow$ : unchanged; $\uparrow$ : increased. ${ }^{\#}$ : 1-month run-in, 6 months each cycle (azithromycin/placebo), 2-month washout period; ${ }^{\uparrow}: 250$ mg for patients $<40$ kg; ${ }^{+}$ intravenous antibiotic courses (instead of days); ${ }^{\text {s: }}$ quality of well being visual analogue score reported; ${ }^{f}$ : physical functioning domain significantly increased (other domains unchanged). 
variable in the four placebo-controlled studies. In the Australian [2] and UK studies [3] there was no weight change between groups. In the USA study [4] there was a significant increase in weight in the AZM-treated patients compared with those receiving placebo. In the French study [5] there was no difference in BMI z-scores between the daily AZM and placebo groups. More studies on the effects of AZM are needed in younger patients with $\mathrm{CF}$.

The microbiological isolates from patients in either arm of the current trial were not extensively studied. The sputum samples were analysed according to established methods of the microbiological laboratories of each hospital. The rate of $S$. aureus isolates at baseline is consistent with that reported for Australian patients with CF [19]. In Australia, the rate of $S$. aureus was 40,37 and $31 \%$ for children aged $0-10 \mathrm{yrs}$, adolescents aged 11-17 yrs and adults with CF, respectively. The current analysis of $S$. aureus isolates demonstrated that five patients developed resistance to AZM during the course of the study. Patients in whom these resistant isolates were found did not have any different outcomes (primary or secondary). $S$. pneumoniae was not isolated and nor was any other upper respiratory tract bacterium acquired during the 6 months; however, it is recognised that such organisms may have been present in small numbers within the normal respiratory flora. Antibiotic resistance is a potential problem with long-term use of AZM [6, 7], and whilst numbers were small in the current study the potential for macrolide resistance of $S$. aureus to develop is has been noted [7]. The clinical impact of this phenomenon requires further study.

There are several limitations of the present study. Detailed microbiological investigation of oropharyngeal bacteriology was not undertaken. The culture methods were those used in routine clinical practice at the five $C F$ centres and had a predictably low yield. The fact that no $S$. pneumoniae isolates were found was, therefore, not surprising. It would be desirable in future studies for more detailed culture methods to examine for changes in macrolide susceptibility of bacteria, such as S. pneumoniae.

Parameters of healthcare utilisation, including episodes of admission for treatment of respiratory symptoms and time to hospitalisation, were reported in the present study. Protocol definitions of respiratory exacerbations were not applied; however, patients were handled equally in both treatment arms. Future long-term studies of the impact of macrolides should include definitions of exacerbations.

The clinical impact of AZM on patients with multi-resistant bacterial infections, such as methicillin-resistant $S$. aureus and B. cepacia complex, has not been formally studied. Patients with these organisms were included in the study but the numbers of patients were small and further studies are required to define the potential role of AZM in patients with multi-resistant bacterial infection. Similarly, the role of AZM in very young patients is uncertain, especially those without evidence of $P$. aeruginosa infection, and additional studies are anticipated [L. Saiman, Columbia University, New York, NY, USA; personal communication]. The current study and the recently published French study included younger children; however, numbers are small and study duration short (6-12 months).
The present study demonstrated that whilst daily and weekly administered azithromycin provide similar outcomes over 6 months, treatment with 1,200 $\mathrm{mg} \cdot$ week $^{-1}$ was associated with increased gastro-intestinal adverse events and daily treatment with $250 \mathrm{mg}$ had modest but more positive effects on growth in children with cystic fibrosis. These latter differences between the two groups are important; future studies involving $250 \mathrm{mg}$ per week would help to determine if these observations are related to the large dose or to an inherent advantage of daily versus weekly administration. There are many other unanswered questions in this area, such as when azithromycin treatment should begin, is the benefit sustained, should administration be continuous or is there an optimal time limit, could other macrolides also be beneficial and could resistance and/or toxicity represent a significant problem? Further studies on these issues are needed to optimise the benefits of azithromycin therapy in cystic fibrosis patients.

\section{ACKNOWLEDGEMENTS}

The authors are grateful to S. Pain, K. Gilshenan and A. Chang (Director of the Mater Research Support Centre; all from Research Support Centre, Mater Health Services, South Brisbane, Australia) for statistical help, and M. Herwig (Dept of Medicine, University of Queensland, Mater Adult Hospital, Queensland, Australia) for clerical assistance. The authors would also like to thank all of the patients and their families, the pharmacy staff at all the hospitals and centre staff who assisted in so many ways with the execution of the study.

\section{REFERENCES}

1 Kudoh S, Uetake T, Hagiwara K, et al. [Clinical effects of low-dose long-term erythromycin chemotherapy on diffuse panbronchiolitis.] Nihon Kyobu Shikkan Gakkai Zasshi 1987; 25: 632-642.

2 Wolter J, Seeney S, Bell S, Bowler S, Masel P, McCormack J. Effect of long term treatment with azithromycin on disease parameters in cystic fibrosis: a randomised trial. Thorax 2002; 57: 212-216.

3 Equi A, Balfour-Lynn IM, Bush A, Rosenthal M. Long term azithromycin in children with cystic fibrosis: a randomised, placebo controlled crossover trial. Lancet 2002; 360: 978-984.

4 Saiman L, Marshall BC, Mayer-Hamblett N, et al. Azithromycin in patients with cystic fibrosis chronically infected with Pseudomonas aeruginosa: a randomized controlled trial. JAMA 2003; 290: 1749-1756.

5 Clement A, Tamalet A, Le Roux E, Ravilly S, Fauroux B, Jais J-P. Long term effects of azithromycin in patients with cystic fibrosis: a double-blind, placebo-controlled trial. Thorax 2006; 61: 895-902.

6 Albrich WC, Monnet DL, Harbarth S. Antibiotic selection pressure and resistance in Streptococcus pneumoniae and Streptococcus pyogenes. Emerg Infect Dis 2004; 10: 514-517.

7 Phaff SJ, Tiddens HA, Verbrugh HA, Ott A. Macrolide resistance of Staphylococcus aureus and Haemophilus species associated with long-term azithromycin use in cystic fibrosis. J Antimicrob Chemother 2006; 57: 741-746.

8 Amsden GW, Gray CL. Serum and WBC pharmacokinetics of $1500 \mathrm{mg}$ of azithromycin when given either as a single 
dose or over a 3 day period in healthy volunteers. $J$ Antimicrob Chemother 2001; 47: 61-66.

9 Wildfeuer A, Laufen H, Zimmermann T. Distribution of orally administered azithromycin in various blood compartments. Int J Clin Pharmacol Ther 1994; 32: 356-360.

10 Scaglione F, Rossoni G. Comparative anti-inflammatory effects of roxithromycin, azithromycin and clarithromycin. J Antimicrob Chemother 1998; 41: Suppl. B, 47-50.

11 Wilms EB, Touw DJ, Heijerman HGM. Pharmacokinetics of azithromycin in plasma, blood, polymorphonuclear neutrophils and sputum during long-term therapy in patients with cystic fibrosis. Ther Drug Monit 2006; 28: 219-225.

12 Bell SC, Senini SL, McCormack JG. Macrolides in cystic fibrosis. Chron Respir Dis 2005; 2: 85-98.

13 Gore CJ, Crockett AJ, Pederson DG, Booth ML, Bauman A, Owen N. Spirometric standards for healthy adult lifetime nonsmokers in Australia. Eur Respir J 1995; 8: 773-781.
14 Spirxpert. Become an expert in spirometry. www.spirxpert com/pivotal2.htm Date last updated: January 2007. Date last accessed: June 29, 2007.

15 American Thoracic Society. ATS statement: Snowbird workshop on standardization of spirometry. Am Rev Respir Dis 1979; 119: 831-838.

16 Miller MB, Gilligan PH. Laboratory aspects of management of chronic pulmonary infections in patients with cystic fibrosis. J Clin Microbiol 2003; 41: 4009-4015.

17 Clinical Laboratory Standards Institute. Performance standards for antimicrobial sensitivity testing. 16th Informational Supplement 2006; 26: M100-S16.

18 Havlir DV, Dube MP, Sattler FR, et al. Prophylaxis against disseminated Mycobacterium avium complex with weekly azithromycin, daily rifabutin, or both. N Engl J Med 1996; 335: 392-398.

19 Australian Cystic Fibrosis Data Registry 2003. http:/ / staging. cysticfibrosis.sitepoint.com.au/projects/dataregistry/ Date last updated: 2005. Date last accessed: June 10, 2007. 